\title{
Smart maintenance and inspection of linear assets: An Industry 4.0 approach
}

\author{
Dammika Seneviratne ${ }^{1}$, Lorenzo Ciani ${ }^{3}$, Marcantonio Catelani ${ }^{3}$, Diego Galar ${ }^{1,2}$ \\ ${ }^{1}$ Tecnalia Research and Innovation, Industry and Transport Division, Miñano (Araba) 01510, Spain \\ ${ }^{2}$ Division of Operation and Maintenance Engineering, Luleå University of Technology, Sweden \\ ${ }^{3}$ Department of Information Engineering, University of Florence, via di S. Marta 3, 50139, Florence, Italy
}

\begin{abstract}
Linear assets have linear properties, for instance, similar underlying geometry and characteristics, over a distance. They show specific patterns of continuous inherent deteriorations and failures. Therefore, remedial inspection and maintenance actions will be similar along the length of a linear asset, but because as the asset is distributed over a large area, the execution costs are greater.

Autonomous robots, for instance, unmanned aerial vehicles, pipe inspection gauges, and remotely operated vehicles, are used in different industrial settings in an ad-hoc manner for inspection and maintenance. Autonomous robots can be programmed for repetitive and specific tasks; this is useful for the inspection and maintenance of linear assets.

This paper reviews the challenges of maintaining the linear assets, focusing on inspections. It also provides a conceptual framework for the use of autonomous inspection and maintenance practices for linear assets to reduce maintenance costs, human involvement, etc., whilst improving the availability of linear assets by effective use of autonomous robots and data from different sources.
\end{abstract}

\section{Section: RESEARCH PAPER}

Keywords: linear assets; autonomous robots; inspection; maintenance

Citation: Dammika Seneviratne, Lorenzo Ciani, Marcantonio Catelani, Diego Galar, Smart maintenance and inspection of linear assets: An Industry 4.0 approach, Acta IMEKO, vol. 7, no. 1, article 9, March 2018, identifier: IMEKO-ACTA-07 (2018)-01-09

Section Editors: Paul Regtien, Measurement Science Consultancy, The Netherlands - Lorenzo Ciani, University of Florence, Italy

Received October 30, 2017; In final form January 5, 2018; Published March 2018

Copyright: (C) 2018 IMEKO. This is an open-access article distributed under the terms of the Creative Commons Attribution 3.0 License, which permits unrestricted use, distribution, and reproduction in any medium, provided the original author and source are credited

Corresponding author: Dammika Seneviratne, Diego Galar, e-mail: dammika.ndb@gmail.com, diego.galar@ltu.se

\section{INTRODUCTION}

A linear (or continuous) asset is an engineering infrastructure that usually spans long distances and can be divided into segments, all of which perform the same function but may be subject to different loads and environmental conditions. Typical linear assets include railway lines, roads, pipelines and cables. Modern society relies heavily on linear assets so it is imperative to manage them effectively.

Research on linear asset management has attracted considerable attention from asset management practitioners and academic researchers, although previous research has focused on specific types of linear assets. For example, Ahern and Anandarajah [1] developed a model for prioritising railway transportation infrastructure using weighted integer goalprogramming. Li et al. [2] developed a management information system for mining railway transportation equipment. Brito and Almeida [3] attempted to rank risks for natural gas pipelines based on multi-attribute utility theory.
Their model can incorporate the decision-maker's preferences and behaviours quantitatively. To model the variable condition of pipeline segments along a route, such as the pipeline failure rate, they divided the pipelines into a number of smaller sections. To address failure issues, Cagno et al. [4] conducted a case study to estimate the prior distributions of gas pipeline failures using the Analytical Hierarchy Process (AHP) and a Bayesian approach. Coffen-Smout and Herbert [5] presented an overview of submarine cable management challenges, while Jones and McManus [6] conducted a life-cycle analysis of five different $11 \mathrm{kV}$ electrical power cables. Link [7] studied the renewal costs of motorways in Germany using an econometric analysis but did not explicitly consider the road condition. Finally, Francesco and Leccese [8] analysed electrical powerlines due to the seismic disaster using FMECA approach.

Conventional asset management systems or decision support tools are not suitable for linear assets; their hierarchical asset structure uses parent-child relationships to link system, 
assembly, component, and part type hierarchies and is not suitable for managing linear assets [9]. To address linear asset issues, IBM began to develop a generic approach to dynamic segmentation using existing standards such as ISO 19133 (Information Technology Geographic Information Framework Data Content Standard) [10]. They discussed this idea among several development partners, including infrastructure managers, academics and government authorities, and decided to segregate the assets into linear and non-linear ones, making them more easily controlled by users. The Maximo Linear Asset Manager 7.1 was released after 3 years; it is based on the concept of 'treating linear assets differently than pumps' through the dynamic segmentation of the former [11], [12]. However, this tool does not integrate inspection and maintenance data and decision analysis functions.

Various types of engineering systems have been developed to acquire data on inspections or to perform maintenance tasks. These are mostly semi-automated systems, i.e., robotic applications. The fields of application range from welding rails to inspecting steel bridges, railways or nuclear power plants. In general, robots need to be developed for specific tasks and fields of application, as these define which locomotion principles or adhesion systems are suitable and which dimensions the robot must have.

While the research results up to date are useful for maintenance management decision support, an effective framework for linear asset management inspection and maintenance needs to be developed to integrate field operations into decision support. As this paper argues, this could be generated using the emerging ICT technologies, for instance, big data technologies.

The paper discusses a framework to incorporate inspection data with maintenance data using big data technologies to manage linear assets by means of autonomous robots. The paper is set up as follows. Section 2 discusses current industrial practices; section 3 explains the industrial challenges; section 4 discusses autonomous inspections and maintenance and explains the framework for the autonomous inspection and maintenance of linear assets; section 5 provides conclusions.

\section{CURRENT INDUSTRIAL PRACTICE}

In today's industrial setting, engineering assets are divided into three categories:
1) Non-linear assets: Non-linear assets can be further classified as component based assets, which include production assets such as machines, mobile assets such as vehicles, and fixed physical assets such as manufacturing facilities [8];

2) Linear assets: Linear assets can be defined as engineering structures or infrastructures that often cross a long distance and can be divided into different segments that perform the same function but are subjected to different loads and conditions;

3) Hybrid systems: These comprise a combination of linear and non-linear assets;

The differences between the asset categories are summarised in Table 1.

Linear assets have the following characteristics:

- Linear assets often form networks which consist of a number of 'lines'. These lines are functionally similar, but can have different characteristics due to various construction materials, operational environments or geometric sizes;

- Linear assets may not have a clear physical boundary. For example, a pipeline is often partitioned using technical, jurisdictional and organisational criteria, and different criteria can result in different, perhaps overlapping, 'pipelines' being identified for the same pipeline network;

- Linear assets usually span long distances and are divided into segments. These segments are often virtually defined based on maintenance activities and may vary across maintenance actions. An example is given in Figure 1. Even though linear assets are constructed of basic elements such as individual pipes in a pipeline, the segments are not necessarily the same as the construction elements. Failures, maintenance events and associated costs are usually recorded for segments, not for the whole linear asset;

- Linear assets are usually long-lived. As a result, when conducting reliability analysis, we may have sparse failure records for a few segments only, as the majority of segments have not failed;

- If any single section of a linear asset malfunctions, the entire asset will not function properly.

Table 1. Three asset categories.

\begin{tabular}{|c|c|c|c|}
\hline Category & Example & $\begin{array}{c}\text { System } \\
\text { configuration }\end{array}$ & Characteristics \\
\hline Linear assets & $\begin{array}{l}\text { Roads, railway } \\
\text { tracks, pipelines, } \\
\text { power cables, } \\
\text { canals and } \\
\text { waterways }\end{array}$ & $\begin{array}{l}\text { Tree structure or } \\
\text { networks }\end{array}$ & $\begin{array}{l}\text {-Maintained and renewed in place and in segments } \\
\text {-No clear physical boundary for segments } \\
\text {-All segments normally perform the same function } \\
\text {-Maintenance costs, failures and maintenance and operational events are associated } \\
\text { with segments } \\
\text {-Segments can be dynamic and are usually long lived }\end{array}$ \\
\hline $\begin{array}{l}\text { Non-linear } \\
\text { assets }\end{array}$ & $\begin{array}{l}\text { Pumps, cars, } \\
\text { machine tools }\end{array}$ & $\begin{array}{l}\text { Complex physical } \\
\text { structure }\end{array}$ & $\begin{array}{l}\text {-Installed and replaced as a whole, but maintained at base maintainable unit (BMU) } \\
\text { level, often in workshops } \\
\text {-BMU has clear physical boundaries } \\
\text {-BMUs often play different functions } \\
\text {-Maintenance cost, failures and maintenance and operational events are associated } \\
\text { with BMUs } \\
\text {-BMUs are usually static } \\
\text {-Lifetime varies }\end{array}$ \\
\hline Hybrid assets & $\begin{array}{l}\text { Power plant } \\
\text { boilers, } \\
\text { refrigerators, } \\
\text { refineries }\end{array}$ & $\begin{array}{l}\text { Complex physical } \\
\text { structure, but linear } \\
\text { and nonlinear parts } \\
\text { have a clear boundary }\end{array}$ & $\begin{array}{l}\text {-Installed, maintained and replaced in parts for large assets and as a whole for small } \\
\text { assets } \\
\text {-Linear subsystems and nonlinear subsystems can often be separated }\end{array}$ \\
\hline
\end{tabular}




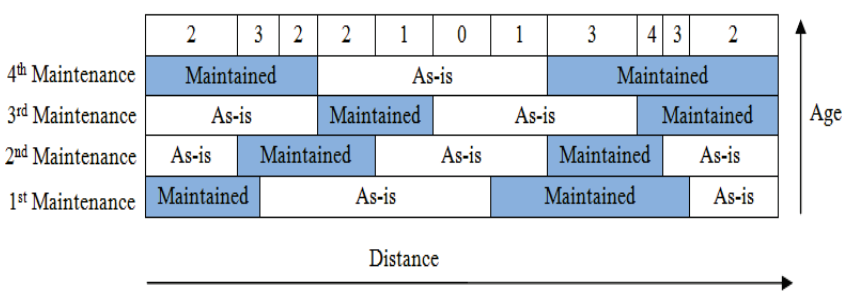

Figure 1. Dynamic maintenance segment boundaries of a linear asset.

These characteristics of linear assets mean that their registry (categorisation), reliability and cost analysis, and maintenance decision modelling methods are different from those of nonlinear assets.

\subsection{Asset Registry Models}

In asset intensive industries where there are strict regulations, the use of a top/down or hierarchical approach to the management of linear assets is both challenging and multifaceted. These types of assets have exclusive requirements that demand a unique asset management approach, often called continuous or linear asset management [13].

In current industrial practice, engineering assets are registered using a hierarchical approach or a 'parent-child' model. Using this model, maintenance attributes, such as maintenance history, repair costs, replacement costs and operational history, are directly linked to individual systems, subsystems, equipment or components. The traditional 'parentchild' hierarchical model works well for most non-linear assets, as there is normally a clearly fixed physical boundary between components. However, a parent-child hierarchy is not suitable for linear assets because of their virtually defined and dynamically changing segments. A network model approach may be useful in addressing these issues [8]. Figure 2 applies the parent-child model to the linear asset hierarchy. The linear reference point (LRP) shown in the Figure 2 is used as a marker schema for the asset. Functional location is the identifier for the functionality of the asset segment. HCA stands for the high consequence areas of the sub system.

\subsection{Data Processing and Segmentation of Linear Assets}

Data grouping plays an important role in the management of linear asset inspection and maintenance. Data grouping demands can be divided into:

- Dividing linear assets into proper segments;

- Correctly grouping segments.

Important data on inspection and maintenance can be gathered by investigating the main factors influencing asset degradation and failure. For water pipelines, for example, these could include pipe materials, soil types, water pressure, age, and pipe diameters.

Segment grouping is critical to scheduling the maintenance

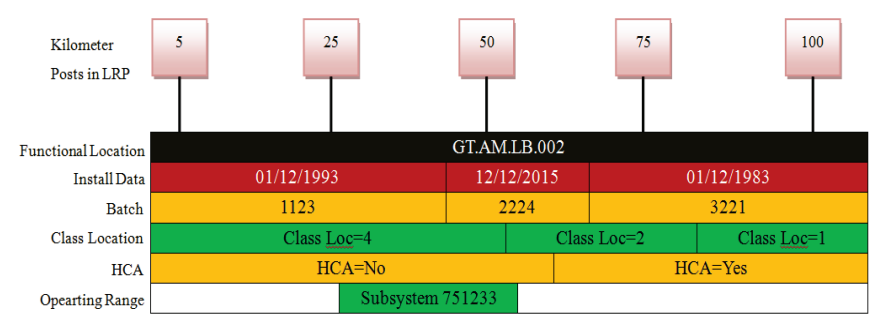

Figure 2. Hierarchical parent-child approach for linear assets. of linear assets. A major challenge is how to group similar but geographically-separated segments, especially with respect to how these groupings influence maintenance decisions and executed maintenance, e.g., sending a repair team out to replace all segments made of the same material and with the same amount of wear in a particular local.

Making maintenance decisions for a linear asset network involves the following:

- Determining which parts of the asset should be renewed together (i.e., a contiguous line or a group of separated but similar segments);

- Determining the best time to renew each part.

The current industrial segmentation of linear assets is generally not suitable for maintenance management statistical analysis, as the assets can have various lengths, and the subdivisions relating to maintenance requirements may overlap. See Figure 3.

\section{INDUSTRIAL CHALLENGE}

\subsection{Challenges of Linear Assets}

The main areas of linear asset use include railways, roads, pipelines, electricity distribution networks, canals and waterways. The major challenges of linear asset management are the following [14]:

1. Structure. Long and straight linear assets are different from up and down ones.

2. Use of GIS. Linear asset management requires data from different sources. Geographic Information Systems (GIS) is one method to acquire data and information. But the reporting of maintenance activities should combine linear asset management data and GIS data.

3. Dynamic segmentation. This is the ability to define changing characteristic features along the length of an asset. For example a segment of pipeline will have changing features along its length. Pipeline is the asset and the features can dynamically change across with piping segment which is maintained.

4. Linear reference methods. The location on a linear asset can be signified in a several ways. The location can be absolute point (start of the segment) or relative to some defined marker such as a kilometre post or distance from a known marker. This referencing is useful to identify faults in the system (Figure 4).

5. Mass changes. Tools are needed to effect mass changes to

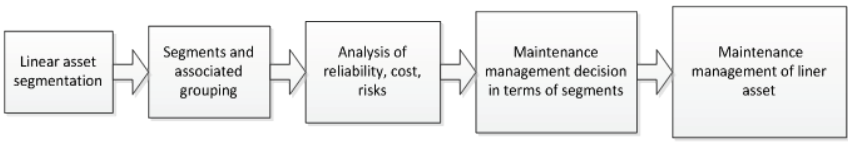

Figure 3. Segmentation of linear assets for maintenance management.

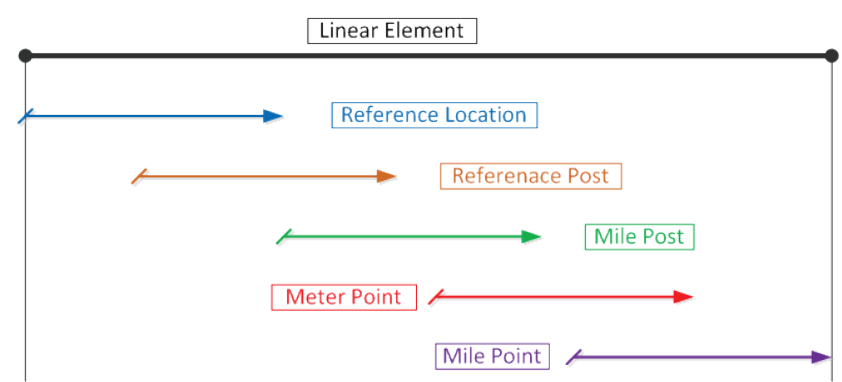

Figure 4. Different methods used to record distance on a linear asset. 
the linear assets under management - this is particularly relevant where costing boundaries change and both the linear assets and point assets need to be updated with new cost collectors.

6. Location information. This can vary depending on the asset type, e.g.:

Roads: Markers, e.g. kilometre posts, lanes, direction, offsets, bridges, tunnels, signs etc.

Rail: Kilometre or chain markers, track number, offsets, switches, crossings, signal etc.

Pipelines: Kilometre points, compressor stations, valves, pig stations etc.

Transmission lines: Substations, traces etc.

Waterways: distribution points, gates, wires, dams, barrages, bridges, culverts, banks etc.

7. Reflection of linear asset: The linear aspect has to be reflected in:

- Inspections

- Condition monitoring

- Maintenance and repair

The maintenance of any linear infrastructure brings many challenges [15]:

1. Modelling linear assets: Modelling is the major challenge as assets are not limited to a single location and may span geographical areas.

2. Assigning non-linear assets to linear assets: Inclusion of non-linear assets within linear assets is difficult as it affects the behaviour of the latter.

3. Parallel networks: With parallel networks, a failure of any one has consequences, such as work stoppage.

4. Intersecting networks: Networks can cross one another, so maintenance carried out on one asset will have an impact on another network. This happens, for example, when road and railways intersect pipelines, waterways and transmission lines.

5. Planned maintenance time: Stoppage for maintenance will result in an increased load on other networks as traffic is diverted; more usage may lead to reductions in performance.

6. Skilled personnel to perform maintenance: Maintaining linear assets requires groups from different departments to work on the same assets and coordination may be difficult.

7. Special equipment for maintenance: Maintaining these assets may require special equipment.

8. Work order planning and execution: The planning and execution of maintenance tasks with multidisciplinary teams is a challenge. 9. Varying load: The modelling of varying loads on linear assets to differentiate the changes in their maintenance needs.

10. Maintenance analytics: In addition to planning and executing the work, other analytics may be required, for example, determining the downtime trend, cost of maintaining the network, allocation of costs to different networks etc.

\subsection{Typical Attributes of a Linear Asset}

- A linear asset has an associated length dimension represented by means of start points and end points or asset length. Components (or features) can be 'installed' along its length.

- Attributes can change frequently along its length.

- It is typically connected with other linear assets to represent an infrastructure network or route.

- A linear asset is subject to dynamic segmentation; multiple sets of attributes can be associated with any portion of an existing linear feature independently of where it begins or ends.
- A linear referencing method is required to describe and locate a position along its length.

- Permanent referencing locations (markers or referencing locations) plus offsets are used to describe an exact position along the linear asset.

\subsection{Challenges in Inspection and Maintenance}

Industrial maintenance service providers find that creating a technical structure to accurately represent the linear assets in their infrastructure network is a highly complex process, making maintenance and inspection strategies difficult to plan and report. Yet maintaining the integrity of these assets ensures performance optimisation and compliance with health safety and environmental (HSE) regulations.

Managing linear assets, such as waterways or rail networks, comes with a unique set of requirements. Asset management functionality is associated with conducting detailed inspections, planning maintenance schedules, devising overhaul schedules and prioritising work to meet safety, budget and customer expectations. To maintain linear assets, asset managers must do the following:

- Split assets into sections based on a definable length breakdown.

- Add records for associated assets along lengths, such as locks, gates, footpaths etc.

- Record multiple ownership records for single assets.

- Import historical maintenance records, images, and any additional data in a dynamic manner.

- Perform on-site, roaming inspections using autonomous devices to record defects, identify issues, add images, log GPS data, and add any other information required.

- Perform maintenance activities through a remotely operated interconnected environment.

\section{AUTONOMOUS INSPECTION AND MAINTENANCE}

Remotely controlled and autonomous inspection and maintenance devices are used in different sectors for different purposes. For instance, the military uses unmanned aerial vehicles for inspection, and offshore oil and gas industries use underwater robots for maintenance.

The following list lists some autonomous or remotely controlled devices used in the inspection and maintenance of linear assets and explains their purpose:

Railways:

- Identification of obstacles and track irregularities using drones.

- Inspection of rail profile, cracks, irregularities, missing components using an autonomous robot vehicle.

- Replacement of missing components, crack welding, etc. using an autonomous maintenance robot vehicle.

Roads:

- Identification of obstacles and damage using drones.

- Inspection of roadway, road alignment, road profile etc. using an autonomous robot vehicle.

- Repair of roadway (placement of asphalt/concrete), repair of pavement, maintenance of embankments, maintenance and cleaning of ditches, etc. using autonomous maintenance robot vehicle.

Canals and Waterways: 
- Identification of debris, obstacles and damages for the infrastructure through drones.

- Inspection of waterway, sidewalls, berm, gates, etc. using an autonomous robot vehicle, both land and water.

- Removal of debris and obstacles, repair of sidewalls, berm, etc. using an autonomous maintenance robot vehicle (both land and water).

Power Lines:

- Identification/inspection of power line damage, insulator defects, tower damage using drones.

- Cleaning of insulators and repair of line damage using an autonomous drone robot vehicle.

\subsection{Smarter Drones}

Unmanned aerial vehicles (UAVs) have attractive features, such as flexibility, adaptability, and a range of payloads. Sensors include high-resolution digital and infrared cameras, Light Detection and Ranging (LiDAR), geographic information systems (GIS), sonar sensors, and ultrasonic sensors; most can be adapted to a UAV platform. A close-up photograph of a structure on an offshore platform, difficult for inspectors to reach, will show maintenance personnel how much corrosion/erosion has built up and suggest the situation of welds and other structural elements.

Drones equipped with forward-looking infrared (FLIR) or ultraviolet sensors can detect hot spots or corona discharge on conductors and insulators, signalling a potential defect or weakness in the component. LiDAR can be integrated with drones to survey a proposed right-of-way, show the infrastructure situation when seismic conditions are changing, or monitor the encroachment of vegetation. There are many more potential uses and the examples are only a small fraction of the possible applications.

At present, UAVs are remotely operated; the next phase of UAV technology will be to deploy 'smarter' machines that can fly autonomously. This technology will allow UAVs to sense and avoid other objects in their path, recognise features or components through various sensors (including cameras) using complex software algorithms such as image processing algorithms, and achieve situational awareness. This advanced technology will foster calculated decision making, such as initiating focused inspections, issuing work orders for repairs, and starting maintenance work with the same robot or another autonomous robot integrated in the system.

In any industry, safety and cost are two of the most significant drivers of operation and maintenance and, thus, are always of high importance. Many industrial work areas are hazardous, so measures must be taken to secure the safety of users. HSE indicators can mitigate the risks, but the situation remains challenging when new technologies are introduced.

For instance, working on energised high-voltage transmission lines, sometimes hundreds of feet up in the air, can make the consequences of a mistake deadly. According to the US Bureau of Labor Statistics, 15 linemen were fatally injured in 2013 as a result of 'exposure to harmful substances or environments' [16].

Unmanned systems have the potential to greatly reduce the amount of risk exposure of the operational workforce. The safety of personnel involved in risky operational tasks can be ensured with this new technology.

\subsection{Autonomous Robots}

Many different robots have been developed to handle various situations on linear assets, buildings, ship hulls, or other human-made structures. However, most are limited to special situations or applications. To execute the desired tasks, autonomous robots, as well as all other technical systems, have to fulfil certain requirements. The requirements and their importance and focus depend on the individual application or tasks. However, we can formulate a general set of requirements as follows:

1. Velocity and mobility: Vehicle speed and dynamics (ability to move) are two main aspects of robot design. Depending on the dimension of the linear asset, it may have to reach a relatively high velocity for sufficiently fast navigation between inspection areas or similar points of action. Another requirement is related to the desired manipulation and positioning capabilities of the system. This includes the precision of locomotion and its trajectory, since some inspection sensors need to be moved in a smooth and continuous way over the surface. The robot may also need to move sideways or to turn $360^{\circ}$ to position sensors or tools. The system dynamics should be able to handle the various terrains and reach all positions of the asset.

2. Payload: Depending on the application, the system must be able to carry payloads of different weights. For example, in the case of steel piping, a payload of $5 \mathrm{~kg}$ or more is mandatory to carry ultrasonic inspection sensors. This requires a much bigger robot than a system which just needs a simple camera with a weight of several hundred grams. In other words, the dimension, adhesion, and motion components of the robot need to be adapted for the application.

3. Reliability and safety: A further important non-functional aspect is the robustness of the system. If the autonomous robot fails frequently during one inspection task, it is not usable in practice. The requirements of reliability and safety include robust hardware, optimal controllers, and methods to detect and handle hazardous situations and to recover from them.

4. Usability: Velocity, manoeuvrability, and the capability of carrying a certain payload are important, but they are only the basis of the general operability of the system. To bring a robotic system into application, it has to be more powerful, more efficient, and less dangerous than common approaches, e.g., in terms of inspection devices. This includes aspects of maintainability and a broad range of other tasks. Therefore, it must be able to carry different payloads (e.g. inspection sensors or tools) depending on the desired task, parts need to be easily replaceable, and the operation must be faster and less complicated than existing approaches. Aspects like energy consumption, weight, or dimension of the system can be important as well. Based on the individual task, a robot developer has to decide which requirements have to be fulfilled and select a suitable locomotion and attraction principle.

\subsection{Autonomous Inspections}

Traditionally, electric power suppliers have inspected power lines for encroaching trees, damage to structures, and deterioration of insulators by having employees traverse the lines on foot and climb the poles. This is time-consuming and arduous, with a considerable element of risk. Now the task is often carried out by crews in manned helicopters using binoculars and thermal imagers to detect the breakdown of insulators. This too is not without hazard.

Recently, trials have tested the use of UAVs to inspect power lines, with considerable success. UAVs offer lower costs, 
do not create a hazard for aircrews, can operate in more adverse weather conditions, and are less obtrusive to neighbouring communities or animals. Hover flight is essential for the inspection task. The UAV carries an electro-optic and thermal imaging payload, the data from which are available in real-time to the operator and recorded. The UAV is automatically guided along the power line within a limited volume of airspace close to the lines using a distance measuring device. An important requirement of UAVs deployed in this role is that they must be flown close to high voltage power lines, that is, within their electromagnetic fields, without adverse effects upon their control system or payload performance.

Oil and gas supplying companies are interested in UAVs for inspection and exploration purposes. UAVs offer a less expensive means of surveying the land where pipelines are installed. They also offer a means of patrolling the pipes to look for disruptions or leaks caused by accidents such as landslides or lightning strikes or for damage caused by vehicles or falling trees. In certain areas of the world, sabotage is not uncommon, so they look for this as well.

UAVs could be used in road and railway inspections and for certain maintenance purposes by traffic infrastructure agencies. In addition to being less expensive to operate than manned aircraft, they are more covert and will avoid distracting drivers.

Irrigation projects, river authorities, and water boards could use UAVs to monitor canals, waterways, pipelines, and rivers. UAVs could be used to monitor reservoirs for pollution or damage or to monitor pipelines for security purposes.

However, the use of UAVs in many of these cases will depend on the approval of the relevant regulatory authorities.

\subsection{Autonomous Maintenance}

Autonomous maintenance activities are mainly associated with robotic applications. Various industries, especially those dealing with high risk activities, are already using remotely operated robots for maintenance activities, for instance, marine repairs (repairs of ships offshore, offshore oil and gas platform maintenance, deep sea pipeline and cable maintenance), oil refinery repairs, nuclear power plant repairs etc. At the moment, because of the limited development of robots for maintenance purposes, complete maintenance cannot be performed in the above mentioned industries.

\subsection{Conceptual Framework}

With autonomous inspection devices and autonomous maintenance robots, a dynamic asset maintenance and management plan can be deployed with the help of big data technologies and available analytics. Right now, industries are using the devices separately for inspection and maintenance; the two have not yet been integrated. By integrating the two operations with the available ICT technologies, the asset maintenance and management process can be automated. The possible architecture for the ICT framework is shown in Figure 5. Moreover, the incorporation of artificial intelligence tools can make the whole process dynamic and autonomous.

Since linear assets have a common behaviour and architecture across their length, the implementation of the concept may reduce costs, ensuring more effective operation and maintenance. The proposed framework is shown in Figure 6.

\section{CONCLUSIONS}

The importance of autonomous inspections and maintenance in linear assets is increasing because many of these

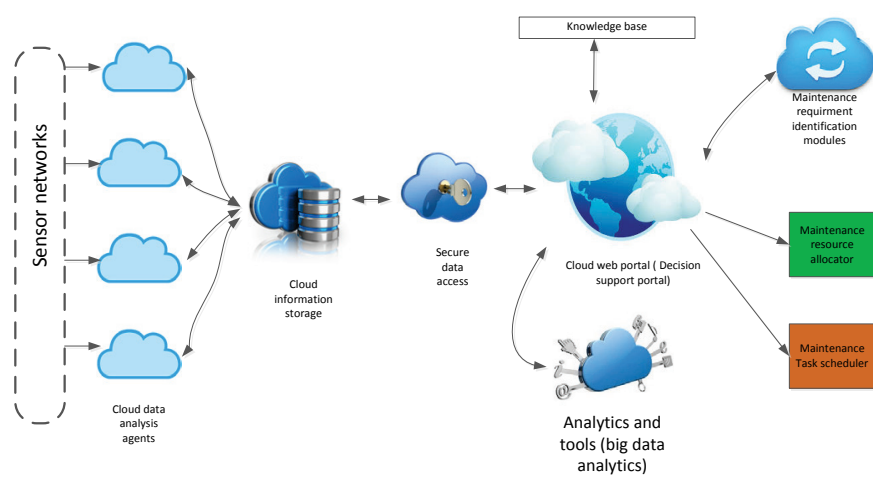

Figure 5. Proposed ICT infrastructure for the autonomous inspection and maintenance of linear assets.

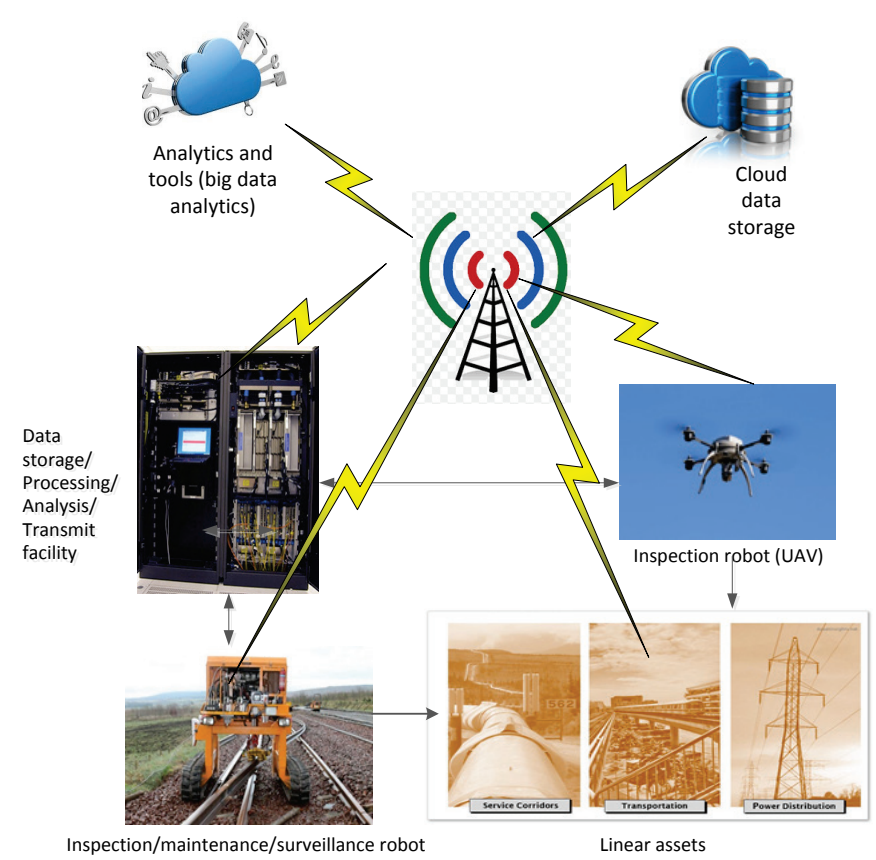

Figure 6. Proposed conceptual framework for autonomous inspection and maintenance of linear assets.

assets are aging and, at the same time, asset managers are struggling to operate effectively and maintain costs. Reliable inspection and maintenance methodologies incorporating new technologies, specifically, the emerging ICT technologies, would facilitate cost effective and efficient asset management.

The industry movement towards new and more sophisticated inspection, condition monitoring, analysis and maintenance technologies, together with the development of autonomous robotics, will provide a platform to maintain large assets (i.e. liner assets) more efficiently. The technology is available but integration remains a key concern.

\section{REFERENCES}

[1] A.Ahern, G. Anandarajah, 'An optimisation model for prioritising transport projects,' In Proc. of the Institution of Civil Engineers-Transport, Thomas Telford Ltd, 2008, vol. 161, no. 4, pp. 221-230.

[2] M.Y.Li, K.Q.Han, X.Y.Zhang, X.L.Li, Y.J.Zhai, W.H.Yu, 'A management information system for mine railway transportation equipment,' Journal of China University of Mining and Technology, 2008, vol. 18, no. 3, pp. 373-376. 
[3] A.J.Brito, A.T.de Almeida, C.M.Mota, 'A multicriteria model for risk sorting of natural gas pipelines based on ELECTRE TRI integrating Utility Theory,' European Journal of Operational Research, 2010, vol. 200, no. 3, pp. 812-821.

[4] E.Cagno, F.Caron, M.Mancini, F.Ruggeri, 'Sensitivity of replacement priorities for gas pipeline maintenance,' In Robust Bayesian Analysis, Springer New York, 2000, pp. 335-350.

[5] S.Coffen-Smout, G.J.Herbert, 'Submarine cables: a challenge for ocean management,' Marine Policy, 2000, vol. 24, no. 6, pp. 441 448.

[6] C.I.Jones, M.C.McManus, 'Life-cycle assessment of $11 \mathrm{kV}$ electrical overhead lines and underground cables,' Journal of Cleaner Production, 2010, vol. 18 no. 14, pp. 1464-1477.

[7] H.Link, 'An econometric analysis of motorway renewal costs in Germany,' Transportation Research Part A: Policy and Practice, 2006, vol. 40, no. 1, pp.19-34

[8] E.De Francesco, F.Leccese, 'Risks analysis for already existent electric lifelines in case of seismic disaster', In Proc. of the 11th International Conference on Environment and Electrical Engineering, EEEIC, 2012, art. no. 6221490, pp. 830-834. DOI: 10.1109/EEEIC.2012.6221490.

[9] Y.Sun, L.Ma, W.Robinson, M.Purser, A.Mathew, C.Fidge, 'Renewal decision support for linear assets,' In Engineering Asset Management and Infrastructure Sustainability, Springer, London, 2012, pp. 885-899.

[10] ISO 19133, 'Geographic information -- Location-based services - Tracking and navigation,' International Organization for Standardisation, Switzerland, 2005.
[11] E. Jones, 'Linear Asset Manager - A Quick History,' [Online]. Available:

https://www.ibm.com/developerworks/community/blogs/a9ba 1efe-b731-4317-9724

a181d6155e3a/entry/linear_asset_manager_a_quick_history18?la $\mathrm{ng}=\mathrm{en}$

[12] Y.Sun, L.Ma, W.Robinson, M.Purser, A.Mathew, C.Fidge, 'Renewal decision support for linear assets,' In Engineering Asset Management and Infrastructure Sustainability, Springer, London, 2012, pp. 885-899.

[13] IBM, 'Better manage your linear assets with IBM Maximo Linear Asset Manager,' [Online]. Available: https://www31.ibm.com/solutions/cn/traveltransportation/pdf/3Better_ma nagemaxio.pdf

[14] Duncan, 'Five reasons why linear assets are different,' [Online]. Available: https://millsone.wordpress.com/2010/03/01/fivereasons-why-linear-assets-are-different/

[15] A. Kaliappan, 'Managing Linear Assets with Oracle EAM,' [Online].

Available: http://www.aioug.org/sangam14/images/Sangam14/Presentati ons/201593_Kaliappan.pdf

[16] D.Schmidt, K.Berns, 'Climbing robots for maintenance and inspections of vertical structures-A survey of design aspects and technologies,' In Robotics and Autonomous Systems, 2013, vol. 61, no. 12, pp. 1288-1305. 